\title{
Blood pressure measurement by oscillometric and auscultatory methods in normotensive pregnant women
}

\author{
Avaliação da pressão arterial pelos métodos oscilométrico e auscultatório em gestantes normotensas
}

Evaluación de la presión arterial por los métodos oscilométrico e auscultatorio en embarazadas normotensas

'Universidade Estadual de Campinas. Campinas, São Paulo, Brazil.

\section{Estefanie Siqueira Vigato' \\ ORCID: 0000-0001-8518-466X \\ José Luiz Tatagiba Lamas' \\ ORCID: 0000-0003-4266-6209}

Vigato ES, Lamas JLT. Blood pressure measurement by oscillometric and auscultatory methods in normotensive pregnant women. Rev Bras Enferm. 2019;72(Suppl 3):162-9. doi: http://dx.doi.org/10.1590/0034-7167-2018-0314

Corresponding Author:

Estefanie Siqueira Vigato

E-mail: estefanievigato@gmail.com

Submission: 05-13-2018

Approval: 09-14-2018

\section{ABSTRACT}

Objective: to compare blood pressure values obtained by auscultatory and oscillometric methods in different gestational periods, considering cuff width. Method: it is a crosssectional and quasi-experimental study approved by the Research Ethics Committee The sample consisted of 108 low-risk pregnant women. Blood pressure measurements were performed in gestational periods of 10-14, 19-22 and 27-30 weeks. Results: The oscillometric device presented values similar to the auscultatory method in systolic blood pressure, but overestimated diastolic blood pressure. Underestimation of blood pressure occurred when using the standard width cuff rather than the correct width cuff in both measuring methods. Conclusion: Verification of brachial circumference and use of adequate cuffs in both methods are indispensable to obtain reliable blood pressure values in pregnant women. We recommend performance of additional studies to evaluate diastolic blood pressure overestimation by the Microlife 3BTO-A.

Descriptors: Pregnancy; Arterial Pressure; Pregnancy-induced Hypertension; Blood Pressure Monitors; Blood Pressure Measurement.

\section{RESUMO}

Objetivo: Comparar os valores de pressão arterial, obtidos pelos métodos auscultatório e oscilométrico em diferentes períodos gestacionais, em função da largura do manguito. Método: Trata-se de um estudo transversal e quase-experimental aprovado pelo Comitê de Ética em Pesquisa. A amostra foi composta por 108 gestantes de baixo risco. As medidas de pressão arterial foram realizadas nos períodos gestacionais de 10-14, 19-22 e 27-30 semanas. Resultados: 0 aparelho oscilométrico apresentou valores similares ao método auscultatório na pressão arterial sistólica, porém superestimou a pressão arterial diastólica. Houve subestimação da pressão arterial ao utilizar o manguito de largura padrão ao invés do manguito de largura correta, nos dois métodos. Conclusão: $\mathrm{A}$ verificação da circunferência braquial e o uso de manguitos adequados nos dois métodos são indispensáveis para obter valores confiáveis da pressão arterial em gestantes. Recomendamos que novos estudos sejam realizados para avaliar a superestimação da pressão arterial diastólica pelo aparelho Microlife 3BTO-A.

Descritores: Gravidez; Pressão Arterial; Hipertensão Induzida pela Gravidez; Monitores de Pressão Arterial; Determinação da Pressão Arterial.

\section{RESUMEN}

Objetivo: Comparar los valores de presión arterial obtenidos por los métodos auscultatorio e oscilométrico en distintos períodos del embarazo, en función del ancho del manguito. Método: Se trata de un estudio transversal y cuasiexperimental aprobado por el Comité de Ética en Investigación. Se analizó una muestra compuesta por 108 embarazadas de bajo riesgo. Las medidas de presión arterial se obtuvieron en los períodos gestacionales de 10-14, 19-22 y 27-30 semanas. Resultados: El aparato oscilométrico presentó valores similares al método auscultatorio en la presión arterial sistólica, sin embargo sobreestimó la presión arterial diastólica. Hubo subestimación de la presión arterial al utilizar el manguito de anchura estándar en lugar del manguito de anchura adecuada, en los dos métodos de medida. Conclusión: La verificación de la circunferencia braquial y el uso de manguitos adecuados en los dos métodos son indispensables para obtener valores confiables de la presión arterial en embarazadas. Recomendamos que se realicen nuevos estudios para evaluar la sobreestimación de la presión arterial diastólica por el aparato Microlife 3BTO-A. Descriptores: Embarazo; Presión Arterial; Hipertensión Inducida en el Embarazo; Monitores de Pression Sanguínea; Determinación de la Presión Sanguínea. 


\section{INTRODUCTION}

According to the World Health Organization, arterial hypertension $(\mathrm{AH})$ affects around $10 \%$ pregnancies worldwide, besides contributing significantly to high rates of neonatal morbidity ${ }^{(1)}$. The Hypertensive Disorders of Pregnancy (HDP) were classified into four types: Chronic arterial hypertension (CAH); Preeclampsia (PE)/eclampsia; $\mathrm{PE}$ superimposed on $\mathrm{CAH}$; and pregnancy-induced hypertension $(\mathrm{PIH})^{(2)}$.

The $7^{\text {th }}$ Brazilian Guideline of Arterial Hypertension brings that AH affects some $7.5 \%$ pregnancies in Brazil, accounting for $20 \%$ to $25 \%$ of all causes of maternal death. Data from the Unified Health System (SUS) show a tendency of stagnation of these levels ${ }^{(3)}$.

The importance of measuring blood pressure (BP) during pregnancy has been emphasized for more than a century, and it is an essential part of prenatal care. Early PE diagnosis has important implications for the treatment and prognosis of both mother and fetus, and this depends on an accurate BP measurement, since its elevation is often the only imminent PE sign ${ }^{(4)}$.

BP decreases from the $1^{\text {st }}$ to the $2^{\text {nd }}$ trimester of gestation. At the $6^{\text {th }}$ week of pregnancy, the values are lower compared to those before pregnancy, and they continue decreasing until the $24^{\text {th }}$ week of pregnancy. Thenceforward, BP gradually increases until delivery, reaching its peak around the $36^{\text {th }}$ week of gestation. This phenomenon is known as "J-curve"(5-8).

$\mathrm{BP}$ can be verified either by direct measurement via intra-arterial catheter or by indirect measurement. Indirect measurement, in turn, encompasses the auscultatory method, which uses a mercury columns device or an aneroid device, and the oscillometric method, using electronic devices ${ }^{(9)}$.

The auscultatory method is still the most practiced in Brazil. However, the mercury manometer has been withdrawn from the market due to problems of environmental contamination, and the aneroid device is the current alternative used in the auscultatory method ${ }^{(10-11)}$.

In order for automatic devices to be used in BP measurement, they must comply with established standards that guarantee their effectiveness. The British Hypertension Society (BHS) ${ }^{(12)}$, the Association for the Advancement of Medical Instrumentation $(\mathrm{AAMI})^{(13)}$ and the European Society of Hypertension (ESH) ${ }^{(14)}$ have developed validation protocols for such pieces of equipment.

Despite the increasing availability of automatic devices to measure BP by patients and professionals and the increased number of validated devices, many researchers are concerned about their indiscriminate use $\mathrm{e}^{(15-17)}$.

Oscillometric devices may perform unreliable BP measurements when used in special groups (diabetic, pregnant, elderly, and arrhythmia patients), since the oscillometric waveform does not present a pattern in all population groups, and may be affected by age and arterial compliance. Therefore, oscillometric devices must be validated independently ${ }^{(11,15)}$.

Even validated, there are oscillometric devices that, in clinical practice, do not meet the precision criteria. They may present failure in their algorithms, influencing measurement results, and may overestimate or underestimate BP in cases, as well as interfering with the diagnosis of $\mathrm{AH}$ and the management of patients at risk ${ }^{(11,16,18-19)}$.
In an attempt to evaluate some of the validation studies limitations, the dabl Educational Trust website was created, which consists of a committee of specialists in BP measurement. According to the website, only five oscillometric devices that have undergone validation study are recommended for use in pregnant women and PE situations ${ }^{(20)}$.

Due to inherent differences between oscillometric and auscultatory methods, pregnancy-puerperium cycle particularities and $\mathrm{HA}$ epidemiological importance in pregnancy, new studies are necessary in this field to evaluate the appropriate use of this type of instrument during pregnancy.

\section{OBJECTIVE}

To compare blood pressure values obtained by auscultatory and oscillometric methods in different gestational periods, considering cuff width.

\section{METHOD}

\section{Ethical Aspects}

The research was approved by the Research Ethics Committee of the University of Campinas (UNICAMP), and Informed Consent Forms were signed by the pregnant women who agreed to participate in the study.

\section{Design, study place and period}

This is a cross-sectional, quasi-experimental study ${ }^{(21)}$ conducted in five Basic Health Units with prenatal care in the countryside of the state of São Paulo, from June to December 2017.

\section{Population or sample; inclusion and exclusion criteria}

Sample size was calculated considering $5 \%$ significance level, $80 \%$ power test and 0.31 effect size, resulting in a sample of 108 low-risk pregnant women aged $\geq 18$ years ( 36 pregnant women per gestational period) $)^{(22-24)}$.

Exclusion criteria were as follows: pregnant women with brachial circumferences $(\mathrm{BC})$ diverging from the available cuffs; multiple pregnancies; pregnant women already diagnosed with $\mathrm{CAH}, \mathrm{PIH}, \mathrm{PE} /$ eclampsia until recruitment, and pregnant women who reported pathologies that could influence BP values, such as chronic kidney disease and arrhythmias.

\section{Study protocol}

The participants were accommodated in a private, quiet and well-lit place. A calibrated aneroid sphygmomanometer, a Littmann adult stethoscope, and cuffs of different widths were used for auscultatory measurement; for oscillometric measurement, a device validated for use in pregnant women (Microlife 3BTO-A) was used. $B C$ was measured with a nondistensible measuring tape.

The oscillometric device was accompanied by a 13-cm cuff, suitable for BC from 22 to $42 \mathrm{~cm}$, according to the instruction manual. In the auscultatory measurement, a 13-cm cuff was considered as standard (standard width cuff - SWC), since it corresponded to the 
same oscillometric cuff width and allowed later comparisons. The correct width cuff (CWC) was calculated according to the formula $\mathrm{CWC}=\mathrm{BC} \times 0.4$, originally proposed by Bordley et al. ${ }^{(25)}$.

Then, the researcher applied a validated questionnaire to characterize the participants, validated after evaluation of five judges. BP measurements were performed by the researcher, trained to perform BP auscultatory measurement according to the recommendations of the $7^{\text {th }}$ al.BGAH ${ }^{(3)}$. The pregnant woman remained resting for five minutes, relaxed in a sitting position with her back against the chair, arm supported at heart level ( $4^{\text {th }}$ intercostal space), slightly bent and absent from clothing, palm facing upwards, legs uncrossed and supported feet ${ }^{(3)}$. The woman was also guided on the procedure to be performed and to remain silent at the time of the measurements.

Subsequently, there was the first raffle, by means of a die, to choose the arm to start the measurements, where even numbers indicated the right arm and odd numbers indicated the left arm. BC measurement was also performed for the arm raffled.

Fourteen measurements were performed in total, where three measurements were made with the aneroid device on the arm raffled, and another three measurements with the aneroid device on the alternate arm. The first measurement was ignored in both arms so that to discard possible white coat hypertension and allow the pregnant woman to adapt to the procedure. The mean of the pairs of measurement of each arm was calculated to determine the arm with the highest pressure value and then perform the subsequent measurements.

After choosing the arm with the highest BP value, eight other measurements were made with alternation of aneroid and oscillometric devices. Two additional raffles were made, also using a die, to choose measurement methods and the cuff width to be used to initiate the measurements (for auscultatory measurement). In the second raffle, even numbers indicated the auscultatory method and odd numbers indicated the oscillometric method; in the third raffle, even numbers indicated the CWC for the BC, and the odd numbers indicated the SWC.

Finally, two measurements were taken with the aneroid device using the CWC (first pair), and two measurements with the aneroid device using the SWC (second pair). For each of these pairs, we had a pair of values obtained by the oscillometric measurement using the SWC indicated by the device. Subsequently, means of all measurement pairs were calculated.

In the auscultatory method (gold standard), systolic blood pressure (SBP) was determined in Korotkoff phase l auscultation, and the diastolic blood pressure (DBP) in phase V. In the cases in which the beats remained audible until level zero, DBP was determined in stage $\mathrm{IV}^{(3)}$. All measurements on the same arm were made with one-minute interval. The values were entered in an Excel spreadsheet and informed to the pregnant woman at the end of all measurements.

The oscillometric device presented no technical issues during data collection.

\section{Analysis of results and statistics}

Analysis of data was made via descriptive and inferential statistics. Analyzes to compare the groups, methods and other qualitative variables regarding BP measurements were performed using "Generalized estimating equations" models (GEE) (26).

For comparisons between the groups regarding quantitative variables, the ANOVA model or nonparametric Kruskal-Wallis test was applied, according to data distribution. To study the associations between qualitative variables, Fisher's exact test ${ }^{(27)}$ was applied only when Chi-square test assumptions ${ }^{(28)}$ were not met.

A 5\% significance level was considered and SAS 9.4 Software was used for analyzes.

\section{RESULTS}

The pregnant women were divided into three groups according to their gestational age: 10 to 14,19 to 22 , and 27 to 30 weeks. More than half (63\%) of participants were less than 30 years old. Among them, $92.5 \%$ were able to answer on family income (in Reais), with a monthly average of $\mathrm{R} \$ 2,402.17$, and the number of minimum wages varied from zero to 11 .

More than 50\% participants cohabited with their partners (74.1\%); reported having completed high school (68.8\%); considered themselves white or brown (76\%), and had external professional activities (57.4\%).

The number of pregnancies ranged from one to six, and primigravidae or secundigravidae accounted for more than $60 \%$ sample. The mean parity $(0.86 \%)$ indicated a low birth rate, and abortion absence was observed in $75.9 \%$ participants.

Regarding the sample, $11.1 \%$ participants used continuous medication; $12 \%$ had HDP previous history; $45.3 \%$ were nulliparous, and $5.6 \%$ were smokers, smoking from one to fifteen cigarettes a day. As for alcohol consumption, 3.7\% used it during pregnancy, from one to four times a month.

There was no significant difference when comparing pregnant women's ages in the three groups. Also, no significant association was found between the groups of pregnant women and age groups, income bracket and number of pregnancies (one gestation and two or more gestations).

It can be seen from Table 1 that the $12-\mathrm{cm}$ cuff was the most used (32.4\%). Smaller cuffs were used in $46.3 \%$ participants, and the larger ones were used in $21.3 \%$. An overall mean of $28.8 \mathrm{~cm}$ of BC was observed, with no significant difference between the groups.

There were no significant differences when comparing blood pressure levels between measurement methods according to income bracket, ethnic groups and schooling.

With regard to SBP, means of auscultatory and oscillometric methods were close but with a significant difference, regardless of cuff width. There was greater variation between the means of the auscultatory method with the SWC and the CWC, also significant ( $p<0.0001)$, indicating BP underestimation when using the SWC instead of the CWC (Table 2).

DBP means obtained with the oscillometric device presented higher values than those obtained with the auscultatory method $(p<0.0001)$, regardless of cuff width. The variations in DBP means between oscillometric and auscultatory methods were $8.4 \mathrm{mmHg}$ with the SWC, and $7.1 \mathrm{mmHg}$ with the CWC. As SBP, DBP was also underestimated when using the SWC ( $p<0.0001)$ (Table 2).

The oscillometric device showed a good performance during BP measurements, since, in 400 measurements, there were 
two attempts of insufflation during the same measurement in only $9.5 \%$ measurements, and only $1.25 \%$ measurements were repeated due to error messages.

Table 1 - Absolute frequency of use of each cuff size in total and according to the group of pregnant women, N=108, Campinas, São Paulo, Brazil, 2018

\begin{tabular}{cccccccccc}
\hline $\begin{array}{c}\text { Cuff } \\
\text { Width (cm) }\end{array}$ & \multicolumn{2}{c}{ Group 1 } & \multicolumn{2}{c}{ Group 2 } & \multicolumn{2}{c}{ Group 3 } & \multicolumn{3}{c}{ Total } \\
& \% & $\mathbf{n}$ & $\%$ & $\mathbf{n}$ & $\%$ & & (Total) & C \\
\hline 9 & 1 & 2.80 & 1 & 2.80 & 2 & 5.60 & 4 & 3.70 & 3.70 \\
10 & 6 & 16.70 & 9 & 25.00 & 3 & 8.30 & 18 & 16.70 & 20.40 \\
11 & 13 & 36.10 & 7 & 19.40 & 8 & 22.20 & 28 & 25.90 & 46.30 \\
12 & 7 & 19.40 & 13 & 36.10 & 15 & 41.70 & 35 & 32.40 & 78.70 \\
13 & 7 & 19.40 & 4 & 11.10 & 5 & 13.90 & 16 & 14.80 & 93.50 \\
14 & 1 & 2.80 & 2 & 5.60 & 2 & 5.60 & 5 & 4.60 & 98.10 \\
15 & 1 & 2.80 & 0 & 00.00 & 1 & 2.80 & 2 & 1.90 & 100.00 \\
\hline
\end{tabular}

Note: C: cumulative percentage

Table 2 - Comparison of systolic and diastolic blood pressure values of the studied sample according to the method and cuff used, represented by the mean [standard deviation (SD)], minimum, median, maximum values and significance level ( $p$ value), N = 108, Campinas, São Paulo, Brazil, 2018

\begin{tabular}{cccccccc}
\hline Variable & Methods & Cuff & Mean (SD) & Minimum & Median & Maximum \\
\hline $\begin{array}{c}\text { Systolic } \\
\text { blood }\end{array}$ & AUSC & SWC & $101.32^{*+}(8.09)$ & 82.00 & 100.50 & 120.00 \\
pressure & OSC 1 & SWC & $102.57(8.42)$ & 79.00 & 102.00 & 122.00 \\
& AUSC & CWC & $104.81^{\ddagger}(7.55)$ & 86.00 & 105.00 & 127.00 \\
& OSC 2 & SWC & $102.93(9.02)$ & 78.00 & 103.00 & 123.00 \\
Diastolic & AUSC & SWC & $55.45^{*+}(9.53)$ & 30.00 & 56.00 & 77.00 \\
blood & OSC 1 & SWC & $63.89(7.13)$ & 40.00 & 63.00 & 82.00 \\
pressure & AUSC & CWC & $57.19^{\ddagger}(8.55)$ & 38.00 & 58.50 & 78.00 \\
& & SWC & $64.31(6.60)$ & 47.00 & 63.00 & 80.00
\end{tabular}

Note: SWC: standard width cuff; CWC: correct width cuff; AUSC: auscultatory method; OSC: oscillometric method; *significant difference between auscultatory method with CWC and auscultatory method with SWC ( $p<0.0001$, GEE model); + significant difference between auscultatory method with SWC and oscillometric 1 ( $p<0.025$, GEE model); $\neq$ significant difference between auscultatory method with CWC and oscillometric 2 ( $p<0.002$, GEE model).

Table 3 - Comparison of diastolic blood pressure values according to the group of pregnant women, device and cuff used, presented by the mean [standard deviation (SD)], minimum, median, maximum values and significance level ( $p$ value), $\mathrm{n}=36$ per group, Campinas, São Paulo, Brazil, 2018

\begin{tabular}{ccccccc}
\hline Group Method & Cuff & Mean (SD) & Minimum & Median & Maximum \\
\hline \multirow{2}{*}{1} & AUSC & SWC & $98.86^{*+}(8.00)$ & 84.00 & 98.50 & 119.00 \\
& OSC 1 & SWC & $101.14(7.28)$ & 83.00 & 101.00 & 122.00 \\
& AUSC & CWC & $102.06(7.60)$ & 86.00 & 100.50 & 119.00 \\
& OSC 2 & SWC & $101.58(8.62)$ & 86.00 & 101.00 & 123.00 \\
2 & & & & & \\
& AUSC & SWC & $101.14^{*}(8.18)$ & 82.00 & 101.00 & 118.00 \\
& OSC 1 & SWC & $101.53(8.98)$ & 79.00 & 103.00 & 117.00 \\
& AUSC & CWC & $105.25^{\ddagger}(7.26)$ & 92.00 & 104.50 & 117.00 \\
& OSC 2 & SWC & $101.72(9.16)$ & 78.00 & 102.50 & 121.00 \\
3 & & & & & & \\
& AUSC & SWC & $103.97^{*}(7.46)$ & 90.00 & 103.00 & 120.00 \\
& OSC 1 & SWC & $105.06(8.57)$ & 90.00 & 103.00 & 122.00 \\
& AUSC & CWC & $107.14(7.09)$ & 95.00 & 106.50 & 127.00 \\
& OSC 2 & SWC & $105.47(8.97)$ & 89.00 & 105.50 & 123.00
\end{tabular}

Note: SWC: standard width cuff; CWC: correct width cuff; AUSC: auscultatory method; OSC: oscillometric method; *significant difference between auscultatory method with CWC and auscultatory method with SWC ( $p<0.0001$, GEE model); $\uparrow$ significant difference between auscultatory method with SWC and oscillometric 1 ( $p<0.0403$, GEE model); $\neq$ significant difference between auscultatory method with CWC and oscillometric 2 ( $p<0.0002$, GEE model).
In the oscillometric method, mean, minimum, median and maximum values were close in SBP and DBP, indicating a uniform behavior of the device (Tables 2, 3 and 4).

In the auscultatory method, the three groups presented a significant difference in SBP when comparing the CWC with the SWC. Again, the use of the SWC led to BP underestimation. SBP means with the CWC were superior to the others. However, only Group 2 presented a significant difference when comparing the auscultatory method with the CWC and the oscillometric method (Table 3).

There was no significant difference between the auscultatory method with the CWC and the oscillometric method in Group 1. However, in the same group, the difference between the auscultatory method with the SWC and the oscillometric method was significant. It may be associated with a possible BP underestimation caused by the use of the SWC in the auscultatory method, but this phenomenon was not evidenced in Groups 2 and 3 (Table 3).

In the three groups, DBP means obtained with the oscillometric device presented higher values ( $p<0.0001$ ) than those obtained with the auscultatory method, regardless of cuff width. Groups 1 and 2 also presented significant differences $(P<0.0001)$ in DBP when comparing the auscultatory method with the CWC and the SWC, again indicating BP underestimation associated with the use of the SWC. Only Group 3 did not present this significant difference (Table 4).

There was DBP overestimation by the oscillometric method in 4.64 $\mathrm{mmHg}, 7.41 \mathrm{mmHg}$ and $9.30 \mathrm{mmHg}$, respectively, in Groups 1,2 and 3, when compared to the auscultatory method with the CWC (Table 4).

Table 4 - Comparison of diastolic blood pressure values of the studied sample according to the group of pregnant women, method and cuff used, presented by the mean [standard deviation (SD)], minimum, median, maximum values and significance level ( $p$ value), $\mathrm{n}=36$ per group, Campinas, São Paulo, Brazil, 2018

\begin{tabular}{ccccccc}
\hline Group & Method & Cuff & Mean (SD) & Minimum & Median & Maximum \\
\hline \multirow{2}{*}{1} & AUSC & MLP & $58.47^{* \dagger}(9.07)$ & 40.00 & 60.00 & 77.00 \\
& OSC 1 & MLP & $64.47(6.26)$ & 51.00 & 64.50 & 81.00 \\
& AUSC & MLC & $60.39^{\ddagger}(7.47)$ & 41.00 & 60.50 & 78.00 \\
& OSC 2 & MLP & $65.03(6.64)$ & 52.00 & 64.50 & 80.00 \\
\multirow{4}{*}{2} & & & & & \\
& AUSC & MLP & $53.86^{*}(8.32)$ & 35.00 & 54.50 & 69.00 \\
& OSC 1 & MLP & $62.94(7.03)$ & 40.00 & 63.00 & 82.00 \\
& AUSC & MLC & $56.31^{\ddagger}(7.20)$ & 41.00 & 56.00 & 69.00 \\
& OSC 2 & MLP & $63.72(5.67)$ & 52.00 & 63.00 & 76.00 \\
& & & & & & \\
3 & AUSC & MLP & $54.03+(10.58)$ & 30.00 & 54.00 & 76.00 \\
& OSC 1 & MLP & $64.25(8.08)$ & 49.00 & 63.00 & 80.00 \\
& AUSC & MLC & $54.89^{\ddagger}(9.94)$ & 38.00 & 54.00 & 76.00 \\
& OSC 2 & MLP & $64.17(7.49)$ & 47.00 & 63.00 & 80.00
\end{tabular}

Note: SWC: standard width cuff; CWC: correct width cuff; AUSC: auscultatory method; OSC: oscillometric method; * significant difference between auscultatory method with CWC and auscultatory method with SWC ( $p<0.004$, GEE model); + significant difference between auscultatory method with SWC and oscillometric 1 ( $p<0.0001$, GEE model); $\neq$ significant difference between auscultatory method with CWC and oscillometric 2 ( $p<0.0001$, GEE model).

When comparing the convergent cuff widths (closest to the SWC), mean differences were smaller, and when comparing the divergent cuffs widths, mean differences were greater. Table 5 shows the comparisons of SBP and DBP differences between the groups of cuffs that would be appropriate for the BC, and the measurement methods that showed significant difference. Cuffs 9 and 10 were put together to allow comparison, since the number of users for each one was lower. 
Table 5 - Mean difference and confidence interval $(95 \% \mathrm{Cl})$ of differences in blood pressure values comparing standard width cuffs with those appropriate for brachial circumference, depending on the method and cuff width used, (13 - 9 or 10: $n=22 ; 13-11: n=28)$, Campinas, São Paulo, Brazil, 2018

\begin{tabular}{cccccc}
\hline Variable & Method & Cuff used & Comparison & MD & $\mathbf{9 5 \% C l}$ \\
\hline \multirow{2}{*}{ SBP } & AUSC & SWC & $13-9$ ou 10 & 8.37 & $3.33-13.41$ \\
& AUSC & SWC & $13-11$ & 5.76 & $1.50-10.02$ \\
& OSC 1 & SWC & $13-9$ ou 10 & 8.74 & $2.57-14.92$ \\
& OSC 2 & SWC & $13-9$ ou 10 & 9.20 & $2.95-15.45$ \\
DBP & AUSC & SWC & $13-9$ ou 10 & 9.44 & $3.71-15.17$ \\
& OSC 1 & SWC & $13-9$ ou 10 & 5.34 & $0.66-10.01$ \\
& & SWC & $13-9$ ou 10 & 5.93 & $2.16-9.70$
\end{tabular}

Note: SWC: standard width cuff; AUSC: auscultatory method; OSC: oscillometric method; MD: mean difference; mixed model was applied.

It should be noted that the largest mean differences, both of SBP and of DBP, were found when comparing the groups of cuffs $9 / 10$ and cuff 13 in the auscultatory methods with the SWC and oscillometric method. These results reinforce the underestimation tendency associated with the use of the SWC.

When analyzing blood pressure levels, the pregnant women were classified as normotensive ( $\mathrm{BP}<120 \times 80 \mathrm{mmHg}$ ) or prehypertensive ( $\mathrm{PH}$ ) ( $\mathrm{SBP} \geq 120$ and/or $\mathrm{DBP} \geq 80 \mathrm{mmHg})$, depending on measurement methods and cuff width. The auscultatory method with the CWC classified three pregnant women as $\mathrm{PH}$, as they presented SBP $\geq 120 \mathrm{mmHg}$. Only one of them was considered $\mathrm{PH}$ by the auscultatory method with the SWC, and two of them were classified as $\mathrm{PH}$ by the oscillometric method.

The oscillometric method considered nine pregnant women as $\mathrm{PH}$. Four of them were considered $\mathrm{PH}$ due to their SBP $\geq 120$ $\mathrm{mmHg}$; and two of them were also classified as $\mathrm{PH}$ by the auscultatory method with the CWC. The oscillometric method considered five pregnant women as $\mathrm{PH}$ due to a DBP $\geq 80 \mathrm{mmHg}$, and any of them was considered as PH by the auscultatory method.

SBP means of the primigravidae $(n=46)$ were higher than in the women with two or more pregnancies $(n=65)$, regardless of measurement methods. However, only the auscultatory method with the CWC identified a significant difference. This difference was not found when DBP was analyzed.

When comparing the differences in pressure values between the three groups of pregnant women, it was found that in SBP, the means of Group 1 were lower than those of the other groups, and those of Group 2 were lower than those of Group 3. Regardless of the measurement method, the largest mean differences in SBP were identified when comparing Groups 1 and 3. These differences were significant when we compared oscillometric and auscultatory methods with the SWC and the CWC. When analyzing DBP, regardless of the measurement method, Group 1 presented the largest mean differences, reaching significance when comparing Groups 1 and 2 using the auscultatory method, regardless of cuff width. We also found statistically significant differences between Groups 1 and 3 only in the auscultatory method with the CWC.

\section{DISCUSSION}

$\mathrm{BP}$ reliable measurement during gestation is essential for prenatal monitoring, contributing to the diagnosis and early intervention in HDP situations. The main finding of this work is that the BP of the pregnant women who composed the sample was underestimated when using the SWC instead of the CWC, regardless of the measurement method.

The oscillometric device used in this study was recommended for clinical use in the adult population, in pregnant women and in PE situations, after undergoing three validation studies following the $\mathrm{BHS}$ protocol, but it was not recommended for use in severe $\mathrm{PE} \mathrm{E}^{(29-31)}$.

When comparing oscillometric and auscultatory methods with the CWC, we found a SBP mean difference close to $2 \mathrm{mmHg}$, therefore without clinical relevance. However, the mean difference in DBP was up to $7.12 \mathrm{mmHg}$, and may be considered of clinical relevance because the oscillometric method overestimated more than $5 \mathrm{mmHg}$. Nevertheless, this difference, greater than $5 \mathrm{mmHg}$, is considered acceptable by the $\mathrm{BHS}$ protocol ${ }^{(8)}$. This value is close to that used by the ESH protocol, which accepts a maximum of $4 \mathrm{mmHg}$ of difference between the values observed by the researchers responsible for the auscultatory measurement ${ }^{(14)}$.

The application of the AAMI protocol (2003) to another validated device revealed a SBP mean difference of 8.54 (SD 9.38), and a DBP mean difference of 4.21 (SD 7.88). These SBP values pointed to the inadequacy of the device for use in emergency and urgency units ${ }^{(11)}$. When analyzing these values according to the latest AAMI protocol(13), DBP also does not fall within the acceptable range (considering only its SD).

The use of the correct cuff in BP measurement is another extremely relevant issue, since using a cuff with width or length inappropriate for the BC causes BP erroneous variation in agreement with the magnitude of the inadequacy. The appropriate cuff width should correspond to $40 \%$ BC, and the appropriate length to $80 \%$ BC. Studies have shown that BP is underestimated when using a cuff which is larger than the appropriate $\mathrm{BC}$, and overestimated when using a cuff smaller than the appropriate $\mathrm{BC}^{(32-33)}$.

Finding diversified sizes of cuffs for purchase is an obstacle faced by healthcare institutions. In view of this, we observed difficulties in finding cuffs appropriate for the BC in clinical practice, mainly in the extreme $B C$ range, where small or large cuffs are required. Small cuffs are hardly encountered, and larger cuffs available are generally oversized and their proportions may not correspond to the width and length recommended to the individual's $\mathrm{BC}^{(34)}$.

The data presented here confirm the occurrence of overestimation or underestimation, depending on cuff width (Tables 2, 3, 4 and 5).

In our study, $21.3 \%$ pregnant women had BC above $31.4 \mathrm{~cm}$, and cuffs $\geq 13 \mathrm{~cm}$ were used. A study on 179 pregnant women identified that $15 \%$ had $B C>33 \mathrm{~cm}$ and required cuffs greater than the standard pattern ${ }^{(35)}$. Considering that obesity is a risk factor for HDP development, BP measurement in obese pregnant women should be meticulous because, when using cuffs with inadequate sizes, this error may contribute to HDP under-diagnosis or misdiagnosis $\mathrm{s}^{(1,33,35)}$.

The pregnant women with $\mathrm{BC}$ lower than $28.8 \mathrm{~cm}$ represented $46.3 \%$ sample of this study. In clinical practice, it is observed that in situations where a smaller cuff is needed, professionals end up using the standard width cuff, usually of $12 \mathrm{~cm}$, since, in most cases, the sphygmomanometer cuff adjusts to the arm even though width and length are inadequate. This evidences that health care unities must provide cuffs of different sizes, and professionals should be aware of the correct use of them ${ }^{(34)}$. 
When dividing pregnant women into groups, Group 3 was the only one that did not present a significant difference in DBP when comparing the SWC and the CWC in the auscultatory method (Table 4). Although the groups had close BC means, it was observed that the highest BC mean was in Group 3 (29.31 $\mathrm{cm}$ ), which used cuffs between $12 \mathrm{~cm}$ and $15 \mathrm{~cm}$ in 64\% pregnant women, which possibly made DBP mean when using the CWC closer to the mean when using the SWC.

Although the oscillometric instrument instruction manual recommends a $13-\mathrm{cm}$ cuff for $\mathrm{CB}$ of 22 to $42 \mathrm{~cm}$, we observed that when using this cuff in pregnant women with $\mathrm{BC}$ that indicated smaller cuffs according to the criterion proposed by Bordley ${ }^{(25)}$, the oscillometric device underestimated SBP and DBP values.

In agreement with this study, a survey on 104 pregnant women found that $80 \%$ sample required cuffs of less than $12 \mathrm{~cm}$. They compared the auscultatory method with the SWC and the CWC, evidencing BP underestimation of up to $10 \mathrm{mmHg}$ when using the SWC. They also found that when comparing the numbers obtained with divergent cuffs, the difference between BP means increased $^{(33)}$.

The oscillometric method classified three times more pregnant women as $\mathrm{PH}$ in relation to the auscultatory method with the CWC. In situations of AH management or HDP diagnosis, this BP overestimation may lead to erroneous diagnosis and unnecessary treatment, particularly in pregnant women with $\mathrm{BC}>33$ $\mathrm{cm}$. However, it may facilitate HDP early diagnosis, since four out of the pregnant women classified as $\mathrm{PH}$ by the oscillometric method belonged to Group 1, number equal to those in Group 3. Certainly, we are talking about a medical diagnosis that should be confirmed by additional data.

Over the last 10 years, several oscillometric devices have successfully passed validation protocols, mainly in general population. Nonetheless, few studies have evaluated the accuracy of automatic monitors in specific populations ${ }^{(18)}$.

Two oscillometric devices previously validated in general population passed again the ESH validation protocol in obese individuals. According to the manufacturers' instructions, one of the devices used a cuff suitable for $B C$ of 13.5 to $21.5 \mathrm{~cm}$, and the other devices used a large cuff suitable for BC of 32 to $42 \mathrm{~cm}$. When comparing them with the mercury manometer, both did not reach the minimum criteria established by the protocol(18).

Another study compared the two methods in pregnant women with $\mathrm{BC}>32 \mathrm{~cm}$, following the BHS protocol, using a mercury manometer and an oscillometric device already validated by the same protocol, in pregnant women and PE situations. The oscillometric method has shown to be imprecise, since it overestimated SBP and DBP. The mean differences between SBP and DBP methods were $7.17 \mathrm{mmHg}$ (SD 6.67) and $9.31 \mathrm{mmHg}$ (SD 6.59), respectively. Thus, the device was classified as not recommended for use in pregnant women with $\mathrm{BC}>32 \mathrm{~cm}^{(19)}$.

In view of these findings, the companies involved in the manufacture of oscillometric devices need to pay attention to the interferences of cuff size in oscillometric measurement, besides providing cuffs of different sizes for use in clinical practice.

In this study, BP underestimation by the oscillometric device was found when using the 13-cm cuff in pregnant women with BC that indicated smaller cuffs. Nevertheless, the oscillometric method presented DBP overestimation when compared to the auscultatory method with the CWC. This means that if we use cuffs appropriate for the BC in oscillometric measurements, the mean differences between the methods may be greater and even of clinical significance.

We verified that BP overestimation by oscillometric method has a possible relation with the BC and use of correct cuffs in auscultatory method. When analyzing each group of pregnant women, cuffs between 9 and $11 \mathrm{~cm}$ were used in 56.5\%, 47.2\% and $36.1 \%$ pregnant women in Groups 1, 2 and 3, respectively. DBP overestimation increased by oscillometric method, although the auscultatory method with the CWC has used cuffs close to the SWC, more frequently in Groups 2 and 3, reducing erroneous BP underestimation by oscillometric method with the use of the cuff 13 in pregnant women with BC that indicated smaller cuffs, as it is the case of Group 1.

In addition, in this study, SBP means in primigravidae were higher than in women with two or more pregnancies, regardless of measurement methods. This difference was significant in the auscultatory method with the CWC. According to Rurangirwa et al. (36), the first pregnancy may be an important risk factor for poor hemodynamic adaptation and vascular complications. The researchers analyzed 8,377 pregnant women, identifying that nulliparous women had higher blood pressure levels and greater risks of developing HDP compared to multiparous women.

A gradual increase in SBP from the first to the third trimester was also identified. No pattern was identified in DBP behavior during pregnancy. Some studies also did not identify a fall in BP around the $20^{\text {th }}$ week of gestation, what would be expected by the "J-curve" phenomenon ${ }^{(37-39)}$. Thus, there is still no consensus on changes in blood pressure values throughout pregnancy.

\section{Study limitations}

The main limitation of the study was its transversal nature, which did not allow the monitoring of pregnant women during pregnancy and the relation of the HDP with measurement methods. In view of the results, new studies, preferably longitudinal, are necessary to compare auscultatory and oscillometric methods, with cuffs of different sizes for the two methods, and analyzes on their relation with HDP occurrence.

\section{Contributions to the nursing and health areas or public policy}

The study emphasizes the importance of having several sizes of cuffs in health institutions, in addition to $B C$ verification by the nursing team and other professionals, contributing to reliable BP measurement in pregnant women. It also reaffirms the need for more rigorous criteria in validation protocols, and the importance of new studies to evaluate the performance of oscillometric devices in clinical practice, so that the method is used safely.

\section{CONCLUSION}

The main conclusion of this research is that $B C$ verification and use of cuffs suitable for it, in both methods, are indispensable to obtain reliable BP values in pregnant women. 
The Microlife 3BTO-A device, even validated for use in pregnant women, presented similar blood pressure values for the auscultatory method in SBP, but overestimated PAD. The use of the SWC underestimated SBP and DBP in pregnant women who would require smaller cuffs, appropriate for their $\mathrm{BC}$. In addition, the oscillometric method classified three times more pregnant women as $\mathrm{PH}$ in relation to the auscultatory method. We recommend that additional studies should be performed in order to thoroughly evaluate this DBP overestimation by the Microlife 3BTO-A device, and its possible interference with $\mathrm{AH}$ diagnosis in pregnant women.

\section{REFERENCES}

1. World Health Organization (WHO). WHO recommendations for prevention and treatment of pre-eclampsia and eclampsia [Internet]. Geneva:WHO; 2011 [cited 2018 Mar 15]. Available from: https://apps.who.int/iris/bitstream/handle/10665/44703/9789241548335_eng.pdf; sessionid=A4CBF4569E8FB0D1CA7B2DD33A1BA0BF?sequence $=1$

2. American College of Obstetricians and Gynecologists; Task Force on Hypertension in Pregnancy. Hypertension in pregnancy. Report of the American College of Obstetricians and Gynecologists'Task Force on Hypertension in Pregnancy. Obstet Gynecol. 2013;122(5):1122-31. doi: 10.1097/01.AOG.0000437382.03963.88

3. Sociedade Brasileira de Cardiologia (SBD). 7a Diretriz Brasileira de Hipertensão Arterial [Internet]. Arq Bras Cardiol. $2016 ; 107$ (3 Supl. 3): 1-83 [cited 2018 Mar 20]. Available from: http://publicacoes.cardiol.br/2014/diretrizes/2016/05_HIPERTENSAO_ARTERIAL.pdf

4. Emiija JS, Vladimir J. Prediction of mild and severe preeclampsia with blood pressure measurements in first and second trimester of pregnancy. Ginekol Pol. 2011;82(11):845-50.

5. Grindheim G, Estensen M, Langesaeter E, Rosseland LA, Toska K. Changes in blood pressure during healthy pregnancy: a longitudinal cohort study. J Hypertens. 2012;30(2):342-50. doi: 10.1097/HJH.0b013e32834fob1c

6. Ochsenbein-Kölble N, Roos M, Gasser T, Huch R, Huch A, Zimmermann R. Cross sectional study of automated blood pressure measurements throughout pregnancy. BJOG. 2004;111(4):319-25. doi: 10.1111/j.1471-0528.2004.00099.x

7. Donelle T. Méthodes de mesure de la pression artérielle pendant la grossesse. Presse Med. 2016:7-8(1):622-6. doi: 10.1016/j.lpm.2016.04.013

8. Ishikuro M, Obara T, Metoki H, Ohkubo T, Yamamoto M, Akutsu K, et al. Blood pressure measured in the clinic and at home during pregnancy among nulliparous and multiparous women: the BOSHI Study. Am J Hypertens. 2013; 26(1):141-8. doi: 10.1093/ajh/hps002

9. Kohlmann NEB, Kohlmann Jr O. Histórico e perspectivas da medida da pressão arterial. Rev Hipert. 2011;14(2):5-13.

10. Tibúrcio MP, Torres GV, Enders BC, Tourinho FSV, Melo GSM, Costa IKF. Contextual analysis of the measurement of blood pressure in clinical practice. J Res Fundam Care Online. 2013;5(3):328-36. doi: 10.9789/2175-5361.2013v5n3p328

11. Mansoor K, Shahnawaz S, Rasool M, Chaudhry H, Ahuja G, Shahnawaz S. Automated Versus Manual Blood Pressure Measurement: A Randomized Crossover Trial in the Emergency Department of a Tertiary Care Hospital in Karachi, Pakistan: Are Third World Countries Ready for the Change? Open Access Maced J Med Sci. 2016;4(3):404-9. doi: 10.3889/oamjms.2016.076

12. O'Brien E, Petrie J, Littler W, de Swiet M, Padfield PL, Altman DG, et al. British Hypertension Society Protocol for the evaluation of blood pressure measuring devices [Internet]. J Hypertens. 1993;11(2):43-62 [cited 2018 Mar 20]. Available from: http://www.eoinobrien.org/wpcontent/uploads/2008/08/x.BHS-Protocol.Revision.J-Hypertens-1993.df_.pdf

13. American National Standard Institute (ANSI). Association for the Advancement of Medical Instrumentation (AAMI). ANSI/AAMI/ISO 810602:2013 Non-invasive sphygmomanometers-part2 Clinical investigation of automated measurement type [Internet]. Washington: ANSI, AAMl; 2013 [cited 2017 Nov 08]. Available from: http://my.aami.org/aamiresources/previewfiles/8106002_1306_preview.pdf

14. O'Brien E, Atkins N, Stergiou G, Karpettas N, Parati G, Asmar R, et al. European Society of Hypertension International Protocol revision 2010 for the validation of blood pressure measuring devices in adults. Blood Pressure Monit. 2010;15(1):23-38. doi: 10.1097/MBP.0b013e3283360e98

15. Benmira A, Perez-Martin A, Schuster I, Aichoun I, Coudray S, Bereksi-Reguig F, Dauzat M. From Korotkoff and Marey to automatic noninvasive oscillometric blood pressure measurement: does easiness come with reliability? Expert Rev Med Devices. 2016;13(2):179-89. doi: $10.1586 / 17434440.2016 .1128821$

16. Stergiou GS, Lourida P, Tzamouranis D, Baibas NM. Unreliable oscillometric blood pressure measurement: prevalence, repeatability and characteristics of the phenomenon. J Hum Hypertens. 2009 Dec;23(12):794-800. doi: 10.1038/jhh.2009.20

17. Amoore, JN. Oscillometric sphygmomanometers: a critical appraisal of current technology. Blood Press Monit. 2012;17(2):80-8. doi: 10.1097/ MBP.0b013e32835026b0

18. Azaki A, Diab R, Harb A, Asmar R, Chahine MN. Questionable accuracy of home blood pressure measurements in the obese population Validation of the Microlife WatchBP $3^{\circledR}$ and Omron RS6 ${ }^{\oplus}$ devices according to the European Society of Hypertension-International Protocol. Vasc Health Risk Manag. 2017 Feb 27;13:61-9. doi: 10.2147/VHRM.S126285

19. James L, Nzelu D, Hay A, Shennan A, Kametas NA. Validation of the Omron MIT Elite blood pressure device in a pregnant population with large arm circumference. Blood Press Monit. 2017;22(2):109-11. doi: 10.1097/MBP.0000000000000239

20. Dabl ${ }^{\oplus}$ Educational Trust. Blood Pressure Monitors - Validation, Papers and Reviews [Internet]. 2017 [cited 2017 Feb 01]. Available from: http://www.dableducational.org 
21. Polit FD, Beckt C. Fundamentos de pesquisa em enfermagem: avaliação de evidências para a prática de enfermagem. $7^{\mathrm{a}}$ ed. Porto Alegre: Artmed; 2011.

22. Cohen J. The significance of a product moment rs. In.: Statistical power analysis for the behavioral sciences [Internet]. 2nd ed. New Jersey: Lawrence Erlbaum Associates; 1988. p. 75-108 [cited 2017 Nov 08]. Available from: http://www.utstat.toronto.edu/ brunner/ oldclass/378f16/readings/CohenPower.pdf

23. Faul F, Erdfelder E, Lang AG, Buchner A. G*Power 3: A flexible statistical power analysis program for the social, behavioral, and biomedical sciences. Behav Res Methods. 2007;39(2):175-91. doi: 10.3758/BF03193146

24. Faul F, Erdfelder E, Buchner A, Lang AG. Statistical power analyses using G*Power 3.1: Tests for correlation and regression analyses. Behav Res Methods. 2009;41(4):1149-60. doi: 10.3758/BRM.41.4.1149

25. Bordley J III, Conner CAR, Hamilton WF, Kerr WJ, Wiggers CJ. Recommendations for human blood pressure determinations by sphygmomanometers. Circulation. 1951;4(4):503-9. doi: 10.1161/01.cir.4.4.503

26. Ghisletta P, Spini D. An Introduction to Generalized Estimating Equations and an Application to Assess Selectivity Effects in a Longitudinal Study on Very Old Individuals. J Educ Behav Stat. 2004;29(4):421-37. doi: 10.3102/10769986029004421

27. Pagano M, Gauvreu K. Princípios de Bioestatística. São Paulo: Ed. Thomson; 2004.

28. Mehta CR, Patel NR. A network algorithm for performing Fisher's exact test in r x c contingency tables. J Am Stat Assoc. 1983;78(382):427-34 doi: $10.2307 / 2288652$

29. Cuckson AC, Reinders A, Shabeeh H, Shennan AH. Validation of the Microlife BP 3BTO-A oscillometric blood pressure monitoring device according to a modified British Hypertension Society protocol. Blood Press Monit. 2002;7(6):319-24. doi: 10.1097/01.mbp.0000047142.34024.dd

30. Reinders A, Cuckson AC, Lee JTM, Shennan AH. An accurate automated blood pressure device for use in pregnancy and pre-eclampsia: the Microlife 3BTO-A. BJOG. 2005;112(7):915-20. doi: 10.1111/j.1471-0528.2005.00617.x

31. Nouwen E, Snijder M, van Montfrans G, Wolf H. Validation of the Omron M7 and Microlife 3BTO-A Blood Pressure Measuring Devices in Preeclampsia. Hypertens Pregnancy. 2012;31(1):131-9. doi: 10.3109/10641955.2010.544799

32. James GD, Gerber LM. Measuring arterial blood pressure in humans: Auscultatory and automatic measurement techniques for human biological field studies. Am J Hum Biol. 2018;30(1):e23063. doi: 10.1002/ajhb.23063

33. Oliveira SMJV, Arcuri EAM, Santos JLF. Cuff width influence on blood pressure measurement during the pregnant-puerperal cycle. J Adv Nurs. 2002;38(2):180-9. doi: 10.1046/j.1365-2648.2002.02162.x

34. Ribeiro CCM. Medidas da pressão arterial em gestantes normotensas na posição sentada e em decúbito lateral esquerdo [mestrado] [Internet]. Campinas: Universidade Estadual de Campinas; 2014 [cited 2017 Dec 20]. Available from: http://repositorio.unicamp.br/ bitstream/REPOSIP/283874/1/Ribeiro_CristianeCrispMartins_M.pdf

35. Hogan JL, Maguire P, Farah N, Kennelly MM, Stuart B, Turner MJ, et al. Body mass index and blood pressure measurement during pregnancy. Hypertens Pregnancy. 2011;30(4):396-400. doi: 10.3109/10641955.2010.506233

36. Rurangirwa AA, Gaillard R, Steegers EA, Hofman A, Jaddoe VW. Hemodynamic adaptations in different trimesters among nulliparous and multiparous pregnant women; the Generation R study. Am J Hypertens. 2012;25(8):892-9. doi: 10.1038/ajh.2012.57

37. Metoki H, Ohkubo T, Obara T, Akutsu K, Yamamoto M, Ishikuro M, et al. Daily serial hemodynamic data during pregnancy and seasonal variation: the BOSHI Study. Clin Exp Hypertens. 2012;34(4):290-6. doi: 10.3109/10641963.2012.681086

38. Nama V, Antonios TF, Onwude J, Manyonda IT. Mid-trimester blood pressure drop in normal pregnancy: myth or reality? J Hypertens. 2011;29(4):763-8. doi: 10.1097/HJH.0b013e328342cb02

39. Adamczak JE, Wolf EJ. Maternal blood pressure adaptation in the first trimester of pregnancy. Am J Perinatol. 2010;27(4):339-42. doi: $10.1055 / \mathrm{s}-0029-1243305$ 